\title{
Commissioning and First Running Experiences with the TOP Barrel PID Detector in the Belle II Experiment
}

\section{Oskar Hartbrich* for the Belle II Barrel Particle Identification Group}

University of Hawaii at Manoa, 2505 Correa Road, Honolulu, Hawaii 96822

E-mail: ohartbriehawaii.edu

The Time of Propagation (TOP) detector is a novel Cherenkov barrel particle identification system built for the Belle II detector upgrade based on quartz radiator bars read out by Micro-Channel Plate PMTs. The readout electronics of the TOP system are built around a switched capacitor array waveform sampling ASIC operating at $2.7 \mathrm{GSa} / \mathrm{s}$. Acquired waveforms are processed in real time in the front end electronics, extracting the individual timing of detected photons to better than $100 \mathrm{ps}$.

This contribution presents the current status of commissioning, calibration and operation of the Belle II TOP detector.

Topical Workshop on Electronics for Particle Physics

11 - 14 September 2017

Santa Cruz, California

\footnotetext{
* Speaker.
} 


\section{SuperKEKB and Belle II}

The B factory experiment Belle at the KEKB collider at KEK (Tsukuba, Japan) [1] was built to experimentally investigate $\mathrm{CP}$ violation in the $\mathrm{B}$ system. Belle and $\mathrm{BaBar}$ (a competing experiment at SLAC) ultimately confirmed large mixing induced CP violation, leading to the 2008 Nobel Prize in Physics for Kobayashi and Maskawa, the theorists who predicted the effect [2]. The upgrade of the accelerator and experiment to SuperKEKB and Belle II, will refine the measurements of previous B factories with vastly improved precision.

The upgraded SuperKEKB accelerator will operate at an instantaneous luminosity of $8 \times 10^{35} \mathrm{~cm}^{-2} \mathrm{~s}^{-1}, 40$ times larger than that of KEKB. The increased luminosity puts high demands on the detector electronics and data acquisition systems, and causes higher radiation levels thoughout the whole detector system. In order to cope with these requirements, the Belle II upgrade fully replaces the tracking and particle identification (PID) systems of the Belle detector, with substantial upgrades to the readout electronics and some of the active material in the outer detector parts [3].

\section{The TOP Barrel PID Detector}

The performance requirement for the Belle II barrel PID system is separating kaons from pions in the momentum range of $1 \mathrm{GeV} / \mathrm{c}$ to $4 \mathrm{GeV} / \mathrm{c}$ with an efficiency of $90 \%$. This is challenging in the environment of the high event rates and machine backgrounds present at SuperKEKB. The Time of Propagation (TOP) barrel particle identification system is based on reconstructing the time of flight and Cherenkov angle of incident charged particles. The Cherenkov photons are generated by its sixteen $250 \mathrm{~cm} \times 44 \mathrm{~cm} \times 2 \mathrm{~cm}$ quartz radiator bars. The Cherenkov photons are totally internally reflected until they are detected by arrays of Micro-Channel Plate PMTs (MCPPMTs) mounted on a small expansion prism on one end of each bar, as shown in Figure 1.

The photon arrival time on the sensor plane is the sum of the time of flight of the particle from the interaction point to the quartz bar and the propagation time of the photon inside the bar before it hits the detector. For each event, the distribution of photon arrival times and positions are compared to individually generated probability density functions (PDFs), generating likelihoods for each possible particle hypothesis. An example for the differences in PDFs between an incident pion and kaon of identical trajectory and momentum is given in Figure 1. However, only 15 to 30 photons out of these distributions are detected per event.

\section{TOP Readout Electronics}

Custom readout front end electronics (FEE) were developed for the TOP detector, with the performance goal of $<100 \mathrm{ps}$ time resolution on individual photon arrival times. The signals of all 8192 MCP channels in the system are acquired by custom Ice Ray Sampler ver. X (IRSX) ASICs, which are continuously sampling into a switched capacitor array at $2.7 \mathrm{GSa} / \mathrm{s}$ [4]. The buffer depth of $10 \mu$ s allows the digitisation and data transfer of dynamically determined groups of samples upon arrival of an external trigger without incurring deadtime in the acquisition.

The TOP FEE is organised into 64 subdetector readout modules (SRMs). One SRM is assembled as a board stack of one Standard Control Readout Data (SCROD) controller board and 


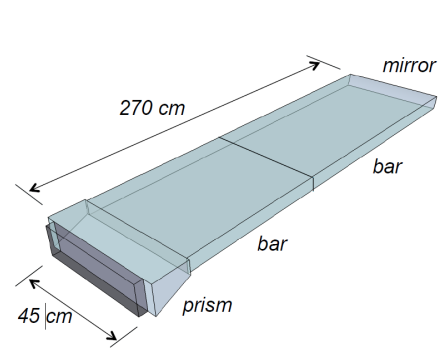

(a) TOP quartz bar

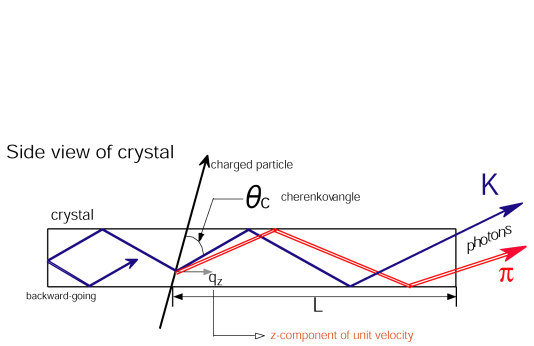

(b) Cherenkov photon propagation

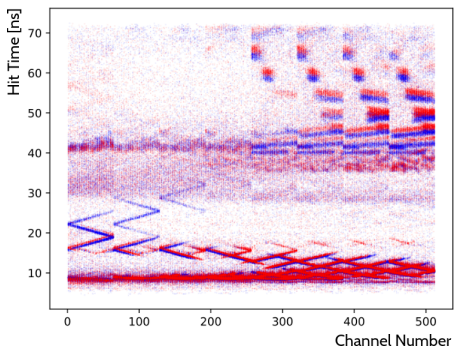

(c) Photon distributions on the TOP sensor plane for simulated pions (blue) and kaons (red). (S. Cunliffe, J. Strube, PNNL)

Figure 1: Schematic view of a TOP quartz bar, illustration of the photon propagation paths through the quartz radiator due to the different angles of radiated Cherenkov photons (angles not to scale), as well as an example of generated PDFs for an incident pion and kaon.

four ASIC carrier boards, hosting four ASICs each, as shown in figure 2. Eight MCP-PMTs are connected directly to the ASIC carrier inputs, for a total of 128 readout channels per SRM.

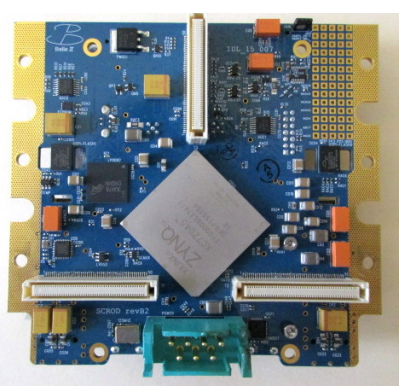

(a) SCROD data concentrator board

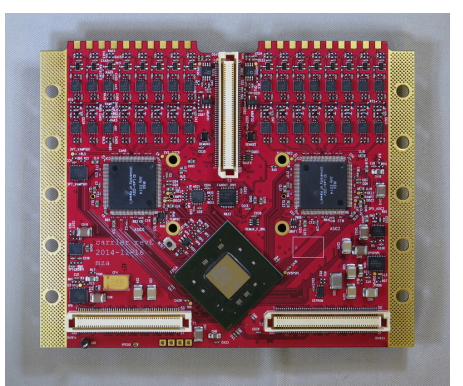

(b) ASIC carrier front end board

Figure 2: Fully assembled TOP front end electronics circuit boards. Each Carrier board directly interfaces the MCP-PMT sensors and integrates four readout ASICs, for a total of 32 readout channels each. Each SCROD board receives the data from a stack of four Carrier boards.

\subsection{Data Reduction in the TOP Frontend Electronics}

As the data bandwidth going out of each SRM is limited during physics running, all signal analysis and data processing of the sampled MCP waveforms is handled online on the Xilinx Zynq Systems on Chip (SOCs) integrated onto the Carrier and SCROD boards. Only the parameters of extracted photon pulses are transferred into the Belle II data stream to be used in the offline reconstruction.

The timing of photon pulses in the digitised waveforms is reconstructed using constant fraction discrimination and template fitting techniques. While the constant fraction discrimination is computationally efficient, its time resolution suffers especially for low amplitude photon pulses. Template fits can improve the extracted time resolution considerably as shown in Figure 3, although with much increased complexity. Efforts towards implementing a template fit inside the Digital Signal Processors (DSPs) of the Zynq SoCs are ongoing. 


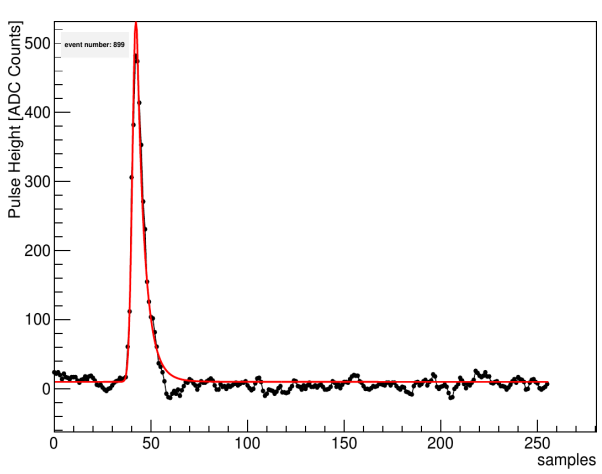

(a) Single photon pulse waveform acquired by the TOP readout electronics with template fit.

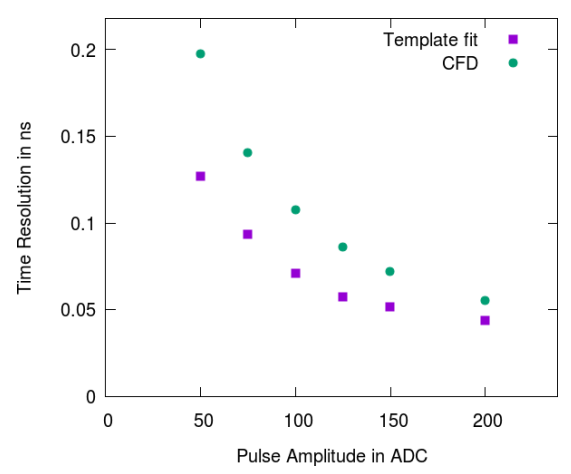

(b) Expected time resolutions of the template fit reconstruction and a constant fraction discrimination method

Figure 3: Example of a template fit reconstruction of single photon pulses recorded by the TOP front end electronics. The template fit offers a considerable improvement in hit time resolution compared to a simple constant fraction technique, especially for low amplitude photon pulses. (T. Weber, University of Hawaii, now RU Bochum)

\section{Current Operation and Global Cosmic Ray Campaign}

The TOP detector has been fully assembled and installed into the Belle II enclosure in Summer 2016 and the whole Belle II detector was successfully rolled into its final position in the beamline in March 2017. Each TOP module has been commissioned and tested with laser pulses and cosmic rays before and after its installation into the TOP enclosure. Combined calibration runs taken with the completed TOP system with laser pulses and direct charge injection have been taken continuously since the completion of the installation. A first global cosmic ray campaign combining the whole outer detector of Belle II, consisting of the central drift chamber (CDC), the electromagnetic calorimeter (ECL), the K-long and muon system (KLM) and the TOP detector was orchestrated during the summer of 2017, in which millions of cosmic ray events were recorded. Example event displays are given in Figure 4.

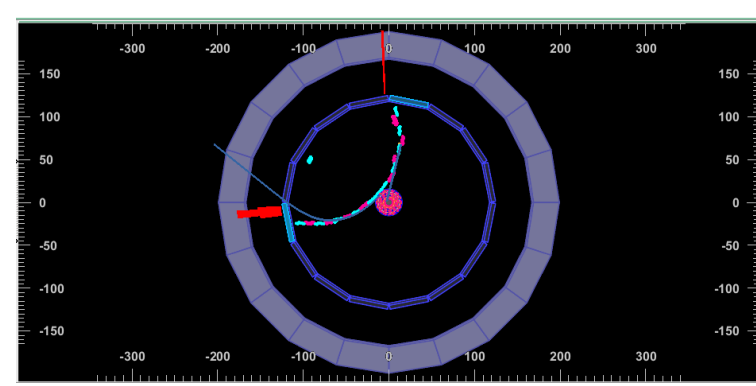

(a) Cosmic muon

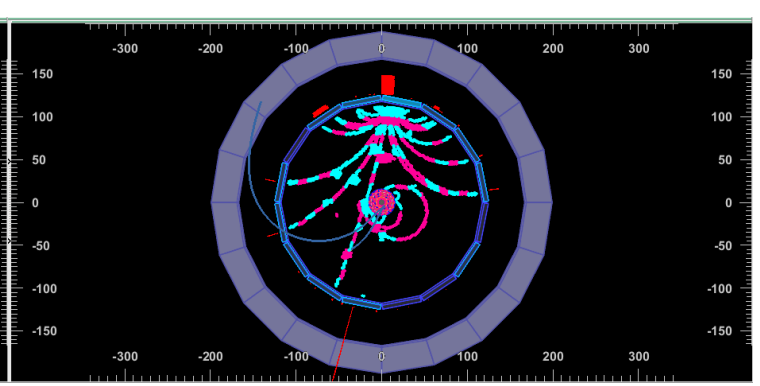

(b) Cosmic shower

Figure 4: Event displays recorded during the global cosmic ray campaign in summer 2017 with the outer Belle II detectors and full solenoid field. Hits in the central drift chamber are drawn separated by stereo layers as pink and teal dots. TOP hits are drawn as teal rectangles. Clusters in the electromagnetic calorimeters are shown as red bars.

Preliminary analyses of the hit efficiency of cosmic muons traversing a TOP module yield 
$>90 \%$ matching efficiency as shown in Figure 5(a).

Using the timing calibration runs periodically recorded with externally injected charge pulses during the global cosmic ray campaign, the intrinsic time resolution of most TOP front end ASICs could be calibrated to around $40 \mathrm{ps}$.

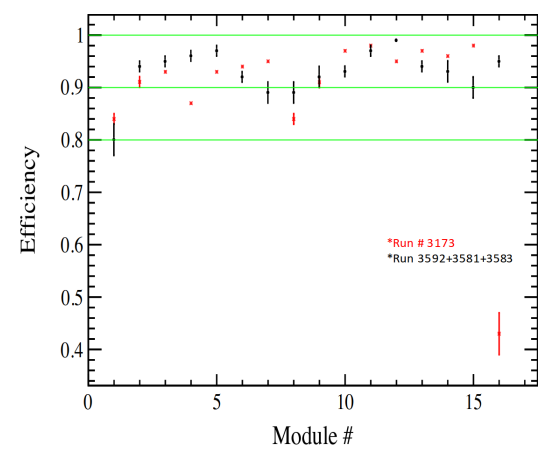

(a) Module hit efficiency (A. Sangal, A. Schwartz, University of Cincinnati)

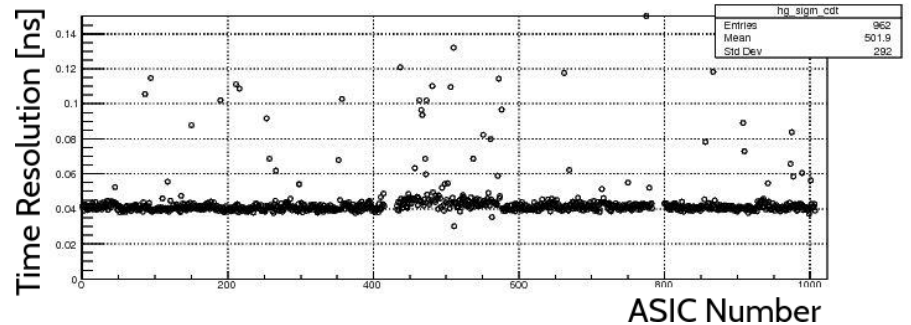

(b) ASIC time calibration (H. Kichimi, KEK IPNS)

Figure 5: Preliminary TOP performance figures during the Belle II global cosmic ray campaign. Most TOP modules show hit efficiencies $>90 \%$. Using in-situ methods, most ASICs can be calibrated to deliver time resolutions of around $40 \mathrm{ps}$.

\section{Summary and Outlook}

The novel TOP barrel PID detector has been successfully constructed and installed into the Belle II detector. The first cosmic ray campaigns and calibration runs with the installed detector system have been very promising. Major system performance aspects have already been studied preliminarily, however further commissioning and firmware development is ongoing.

The SuperKEKB accelerator and the Belle II detector are now preparing for early collisions data-taking with a limited inner tracking system. This "early collisions" phase should start by the end of March 2018. In early 2019, full physics data taking will commence with fully installed inner tracking detectors.

\section{References}

[1] A. Abashian et al. The Belle Detector. Nucl. Instrum. Meth., A479 (2002) 117-232, 2002

[2] M. Kobayashi, T. Maskawa. CP Violation in the Renormalizable Theory of Weak Interaction. Prog. Theor. Phys., 49 (1973) 652-657, 1973.

[3] T. Abe et al. Belle II Technical Design Report. Tech. rep., KEK, 2010

[4] M. Andrew. 128 channel waveform sampling digitizer/readout in the TOP counter for the Belle II upgrade.

Proc. of Sci., TIPP2014, 2014. 